CLINICAL STUDY

\title{
Prospective evaluation of thyroid imaging reporting and data system on 4550 nodules with and without elastography
}

\author{
Gilles Russ $^{1,2}$, Bénédicte Royer ${ }^{1,3}$, Claude Bigorgne ${ }^{1}$, Agnès Rouxel ${ }^{1,2}$, Marie Bienvenu-Perrard ${ }^{1,4}$ \\ and Laurence Leenhardt ${ }^{1,2}$ \\ ${ }^{1}$ Centre of Pathology and Radiology, 14 Avenue René Coty, 75014 Paris, France, ${ }^{2}$ Department of Nuclear Medicine, Pitié-Salpêtrière Hospital, \\ Pierre et Marie Curie University, Boulevard de l'Hôpital, 75651 Paris, France, Departments of ${ }^{3}$ Pathology and ${ }^{4}$ Nuclear Medicine, Cochin Hospital, \\ 27 Rue du Faubourg Saint Jacques, 75014 Paris, France \\ (Correspondence should be addressed to G Russ at Centre of Pathology and Radiology; Email: gilles.russ@wanadoo.fr)
}

\begin{abstract}
Objective: To evaluate prospectively the diagnostic accuracy of the thyroid imaging reporting and data system (TI-RADS) and its interobserver agreement and to estimate the reduction of indications of fineneedle aspiration biopsies (FNABs).

Design: A prospective comparative study was designed.

Methods: In 2 years, 4550 nodules in 3543 patients were prospectively scored using a flowchart and a six-point scale and then submitted to US-FNAB. Results were read according to the Bethesda system. Histopathological results were available for 263 cases after surgery. Sensitivity, specificity, negative predictive value (NPV) and positive predictive value, and accuracy were calculated for the gray-scale score, elastography, and a combination of both methods. Interobserver agreement was calculated using the kappa statistic. The reduction in the number of FNABs was estimated.

Results: When compared with cytopathological results, sensitivity, specificity, NPV, and accuracy were 95.7, 61, 99.7, and $62 \%$ for the TI-RADS gray-scale score; $74.2,91.1,98$, and $90 \%$ for elastography; and $98.5,44.7,99.8$, and $48.3 \%$ for a combination of both methods respectively. When compared with histopathological results, the sensitivity of the gray-scale score, elastography, and a combination of both methods were 93.2, 41.9, and 96.7\% respectively. Interobserver agreement for the six-point scale and the recommendation for biopsy were substantial ( $\kappa$ value $=0.72$ and 0.76 respectively). The reduction in the number of FNABs was estimated to be $33.8 \%$.

Conclusion: The TI-RADS score has high sensitivity and NPV for the diagnosis of thyroid carcinoma. A hard nodule should always be considered as suspicious for malignancy but elastography cannot be used alone. Combination of elastography with gray-scale can be used to improve sensitivity or specificity. Interobserver agreement and decrease in unnecessary biopsies are significant.
\end{abstract}

European Journal of Endocrinology 168 649-655

\section{Introduction}

During 2009, two separate teams $(1,2)$ suggested an interesting thyroid imaging reporting and data system (TI-RADS) derived from the breast imaging reporting and data system (BI-RADS) (3). Initial TI-RADS scores stratified the risk of thyroid nodule malignancy with ultrasound (US) scanning. However, they appeared difficult to apply in daily practice.

With this limitation in mind, our group built a reproducible TI-RADS system that could be used by many institutions. First, we proceeded to perform a retrospective study on 500 nodules (4). The sensitivity, specificity, and odds ratio of each US sign were calculated, and a specific vocabulary and a standardized report were established. A flowchart was developed to easily define the score of a particular nodule. Sensitivity, specificity, and odds ratio of this version of the TI-RADS score were 95, 68, and 40 respectively (95\% CI, 33.7-47.9) (4).
The main goal of this study was to test prospectively the diagnostic accuracy of our TI-RADS score with this flowchart by comparing gray-scale score, elastography, and a combination of both methods with cyto- and histopathological results. The three imaging methods were also intercompared. Secondary objectives were to determine interobserver agreement and to estimate the decrease in unnecessary fine-needle aspiration biopsies (FNABs).

\section{Materials and methods}

\section{Imaging technique and TI-RADS scoring}

Between April 2010 and March 2012, 3543 patients were referred to our specialized thyroid clinic for US-guided FNABs (US-FNAB) of 4550 nodules. US scanning and sono-elastography were performed using 


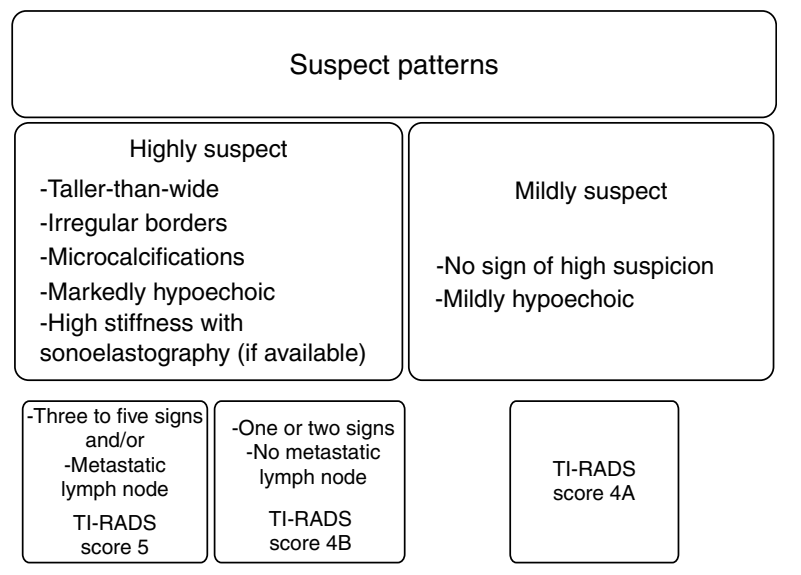

Figure 1 First part of the flowchart designed to score nodules with US. It defines the patterns of nodules suspicious for malignancy. Nodules highly suspicious for malignancy (TI-RADS scores 4B and 5) have at least one of five signs listed in the left column, whereas nodules moderately suspicious for malignancy (TIRADS score $4 \mathrm{~A}$ ) have none of these five signs and are mildly hypoechoic.

a Toshiba Aplio MX scanner (Toshiba Medical Systems Europe, Zoetermer, The Netherlands) with an electronically focused near-field linear probe at $8-18 \mathrm{MHz}$ bandwidth. All nodules were scored with a flowchart (Figs 1 and 2) based on a simplified version of TI-RADS, as previously published (5). TI-RADS scores range from 1 to 5. TI-RADS 1 corresponds to a normal gland, TI-RADS 2 to a benign nodule, and TI-RADS 3 to a highly probable benign nodule (Fig. 3). Suspicion of malignancy can be divided into three categories: TI-RADS 4A (Fig. 4) and 4B (Fig. 5) correspond to low and high suspicion for malignancy respectively, whereas TI-RADS 5 corresponds to a malignant nodule with more than two criteria of high suspicion. Color Doppler assessment was not used because of its poor efficiency and reproducibility $(5,6)$.

Elastography and a combination of gray-scale and elastography were performed on a subset of 1305 nodules. This subset of nodules corresponded mostly to patients that were only referred for FNAB of a single nodule for practical reasons that saved time and simplified data analysis. Elastography was performed using the quantitative elastography module 'real-time elastography' available on the Toshiba Aplio. Light manual compression and decompression were applied to acquire raw data. A series of images representing strain values were generated, and a region of interest was drawn to outline the nodule. A quantitative strain value was calculated. Using receiver operating characteristic (ROC) curve analysis, a threshold was calculated, separating normal and suspect nodules. Qualitative analysis of colored images was not performed because of poor reproducibility (7). For a combination of both methods, nodules with high stiffness were scored at least $4 \mathrm{~B}$, regardless of their gray-scale score, and nodules with low (normal) stiffness had their gray-scale US scores unchanged.

\section{Thyroid FNAB and cytological interpretation techniques}

All 4550 nodules were submitted to FNAB and biopsies were performed using a capillary US-guided FNAB technique with $27 \mathrm{G}$ needles. In most of the cases, only one needle pass was made per lesion. The Bethesda classification was used to interpret smears (8). Cytological specimens were divided into six categories ranging from 1 to 6 , respectively, as follows: nondiagnostic, 91 cases (2\%); benign, 3518 cases $(77.3 \%)$; atypia of undetermined significance, 415 cases $(9 \%)$; suspicious for a follicular neoplasm, 256 cases $(5.6 \%)$; suspicious for malignancy, 130 cases $(2.8 \%)$; and malignant, 140 cases $(3.1 \%)$.

\section{Histopathological results}

Histopathological results were available for 263 cases after surgery for the gray-scale score and for 74 inclusive cases where elastography was performed as well. Surgery was indicated based on cytopathological results (69 malignant, 86 suspect, 89 follicular neoplasm, and nine atypia of indeterminate significance cases) or when the nodule was benign but larger than $3 \mathrm{~cm}$ and causing compressive symptoms (10 cases). There were 130 benign cases: 104 adenomas, 17 adenomatous goiters, and nine atypical adenomas. There were 133 malignant cases: 94 classical papillary carcinomas, 23 follicular variants of papillary carcinoma, seven medullary carcinomas, three poorly differentiated carcinomas, three follicular carcinomas, one oncocytic (Hürthle cell) carcinoma, and two metastases.

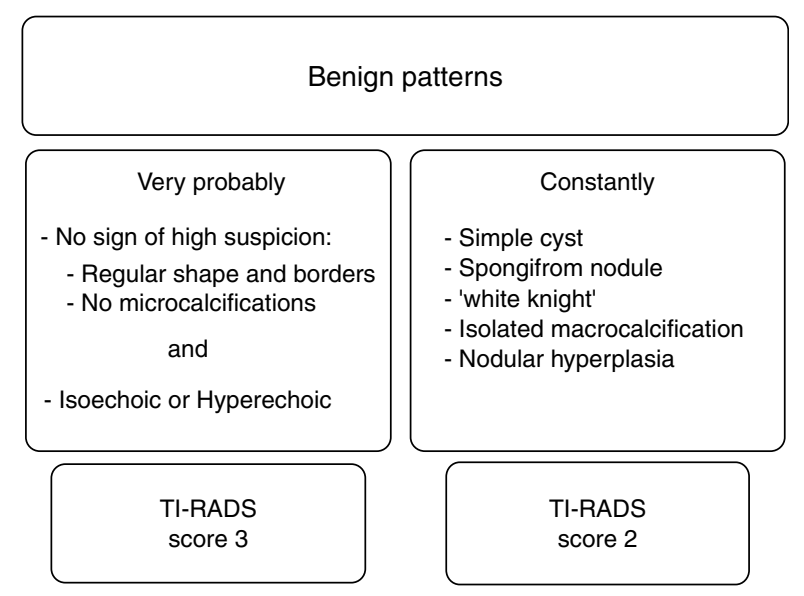

Figure 2 Second part of the flowchart designed to score nodules with US. It defines the patterns of benign nodules. In the context of auto-immune thyroiditis, a white knight pattern is representative of numerous hyperechoic round pseudo-nodules with no halo or central vascularization. Nodular hyperplasia is representative of isoechoic confluent micronodules located within the inferior and posterior portion of one or two lobes, usually avascular and seen in simple goiters. 


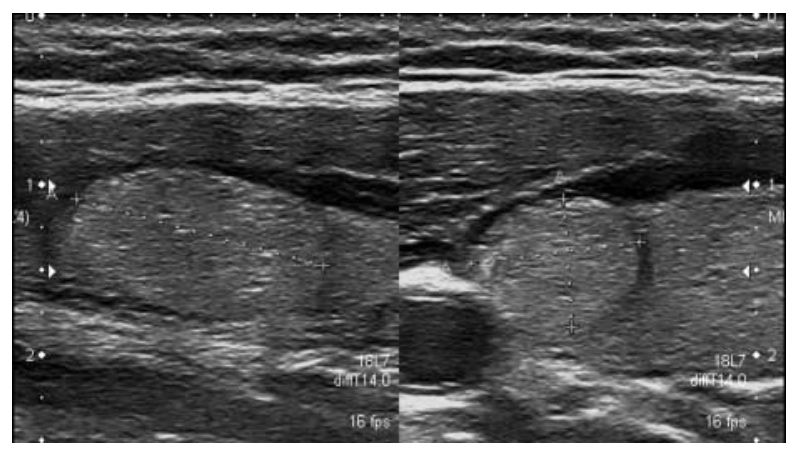

Figure 3 Regular-shaped, round, isoechoic solid nodule with regular borders and shape and no calcifications: TI-RADS 3 .

This nodule was classified as benign by cytopathological analysis.

\section{Assessment of imaging technique efficiency}

Firstly, cytopathological results, excluding nondiagnostic lesions and lesions of indeterminate significance (atypia of undetermined significance, and suspicions of follicular neoplasm and malignancy), were used to compare the TI-RADS gray-scale score, elastography, and a combination of both methods; thus, leaving only Bethesda categories 2 and 6 . This was done because the probability of mistake of these two categories is $<3 \%$ compared with histopathology, in contrast to the underlying diagnostic uncertainties in case of indeterminate or nondiagnostic cytological results. By consequence, 3658 nodules were analyzed with the gray-scale score only, 991 nodules with elastography only, and 991 nodules with a combination of both methods out of a total of 4550 nodules. Secondly, the TI-RADS gray-scale score and elastography were compared with histopathological results, including cytological lesions of indeterminate significance. Sensitivity, specificity, negative predictive value (NPV) and positive predictive value (PPV), and accuracy of the TI-RADS score were calculated. The TI-RADS score was considered exact when its result was benign (score 1,2 , or 3 ) and the final diagnosis after cytology and/or histology was benign or when its result was suspicious for malignancy $(4 \mathrm{~A}, 4 \mathrm{~B}$, or 5$)$ and the final diagnosis was malignant.

\section{Interobserver agreement}

On a subset of 180 consecutive nodules for which patients were referred for both US examination and FNAB, each nodule was successively scored in two different rooms equipped with the same US machine by two different practitioners, the second one having no possibility of knowing the prior score. Agreement was measured by taking into account firstly the complete six-point scale and secondly by grouping scores $4 \mathrm{~A}, 4 \mathrm{~B}$, and 5 . The second approach corresponded to theoretical indications of whether to proceed to FNAB.

\section{Estimation of the decrease in unnecessary FNABs}

We considered that nodules that scored 2 and 3 could be monitored safely without FNAB unless they increase in a proven way. We used previously published data to estimate the number of nodules that were likely to grow $(9,10,11,12)$ and multiplied it by the total number of nodules scored TI-RADS 2 and 3 . We considered $35 \%$ as the most realistic rate of growing nodules and chose to use it for our estimation.

\section{Statistical analysis}

Statistical analysis was performed by Medcalc (MedCalc Software, Ostend, Belgium). ROC curve analysis was used to compare the three imaging techniques and to determine the optimal cut-off value between benign and suspicious nodules. Area-under-the-curve (AUC) and $P$ value were calculated. Differences between sensitivity and AUC for the three imaging techniques were assessed by pairwise comparison using Student's $q$ and Tukey tests. $P$ values $<0.05$ were considered significant. Interobserver agreement was calculated using Cohen's kappa test and Pearson's correlation coefficient.

Approval was obtained from the Institution's Ethic Committee. Complete oral and written information on the purpose and nature of all procedures used was given to every patient, and informed consent was also obtained.

\section{Results}

\section{Demographic data and global results of 4550 nodules by score}

The median age of patients was 54 years (range 14-85). The prevalence of nodules was lower in men (sex ratio 0.22 ).

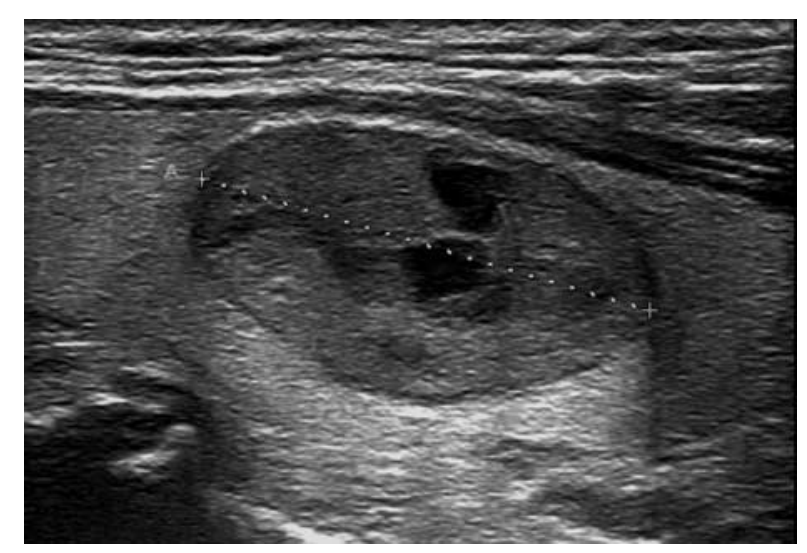

Figure 4 Moderately heterogenous nodule with isoechoic and mildly hypoechoic regions, regular shape and borders: TI-RADS 4A. Mildly hypoechoic is defined as more hypoechoic than the surrounding gland but less than strap muscles. This nodule was classified as a microvesicular adenoma by histopathological analysis. 


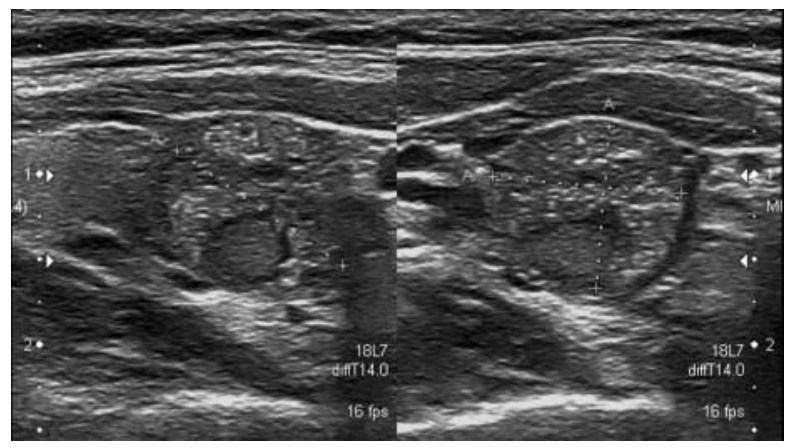

Figure 5 Solid isoechoic nodules with ill-defined borders and microcalcifications: several round, sometimes linear, and tiny punctuations. At the opposite of granular deposits, no US absorption below the punctuations and no hypoechoic microcavities above them are seen, as well as no comet-tail artifacts, TI-RADS 4B. This nodule was cytologically suspicious for malignancy and classified as a papillary carcinoma by histopathological analysis.

The median size of nodules was $17 \mathrm{~mm}$ (range 4-82). For benign nodules, the range was $5-61 \mathrm{~mm}$ and for malignant nodules, it was $4-82 \mathrm{~mm}$. There was no nodule size threshold for indicating FNAB. A total of 252 nodules measured $<10 \mathrm{~mm}$.

Nodules were classified as TI-RADS 2, 3, 4A, 4B, and 5 in $4.2,48.3,44.5,2.7$, and $0.3 \%$ of cases respectively. Distribution of carcinomas among TI-RADS scores was $0,4.3,54,32.8$, and $9.3 \%$ respectively. By cytology, 92\% of TI-RADS scores 2 and 3 were benign, and $1.6 \%$ were suspicious of malignancy or malignant (Bethesda categories 4, 5, and 6). TI-RADS scores $4 \mathrm{~A}, 4 \mathrm{~B}$, and 5 represented $95.7 \%$ of carcinomas (Bethesda 6) and 91.7\% of suspicious lesions (Bethesda 4 and 5).

\section{Diagnostic performance of gray-scale US TI-RADS score compared with cytological results ( 3658 cases)}

When compared with Bethesda categories 2 and 6, sensitivity, specificity, NPV, PPV, and accuracy of the gray-scale TI-RADS score were 95.7, 61, 99.7, and $62 \%$ respectively. PPV was studied for each score separately: $0 \%$ for score $2,0.25 \%$ for score $3,6 \%$ for score $4 \mathrm{~A}, 69 \%$ for score $4 \mathrm{~B}$, and $100 \%$ for score 5 .

\section{Diagnostic performance of elastography and a combination of both methods compared with cytological results (991 cases)}

ROC curve analysis confirmed an optimal cut-off value of 0.045 between stiff and soft nodules, in agreement with a previously published study (13). Values $<0.045$ represented stiff nodules and vice versa. Among 1305 elastographic procedures, six were nondiagnostic because the compression curve was of poor quality. There were 49 true-positive, 861 true-negative, 17 falsenegative, and 64 false-positive cases. The remaining cases represented cytologically undetermined results and were excluded. Sensitivity, specificity, NPV, PPV, and accuracy of elastography were $74.2,91.1,98,37.4$, and $90 \%$ respectively.

Using a combination of both methods, the gray-scale score, which was initially 3 or $4 \mathrm{~A}$, was raised to $4 \mathrm{~B}$ in 85 patients, the nodule being also hard on elastography. There were 65 true-positive, one false-negative (scored 3), 414 true-negative, and 512 false-positive cases. Sensitivity, specificity, NPV, and accuracy were 98.5, $44.7,99.8$, and $48.3 \%$ respectively. Sensitivity of the combination was statistically superior to the gray-scale alone $(P<0.0001)$, but specificity and accuracy decreased $(P<0.0001)$. A comparison of the three methods is shown in Table 1. By ROC curve analysis, AUC was 0.868 (95\% CI, 0.856-0.879; $P<0.0001)$ for gray-scale, 0.834 (95\% CI, $0.810-0.857 ; P<0.0001)$ for elastography, and 0.914 (95\% CI, 0.895-0.931; $P<0.0001)$ for a combination of both methods. The only significant difference between AUCs was between elastography alone and a combination of both methods $(P=0.01)$. PPV was also studied for each score separately (Table 2).

\section{Comparison of TI-RADS score, cytological and histopathological results in the operated group}

The total number of carcinomas was 204 , a rate of $4.5 \%$ (204/4550). They corresponded to 139 cases asserted by cytological analysis (among them 68 cases were confirmed by histopathology after surgery) and to 65 cases diagnosed after surgery and initially classified with the Bethesda system as category 3, 4, or 5. Results are shown in Table 3. The sensitivity of the TI-RADS

Table 1 Comparison of the clinical efficiency of TI-RADS grey scale score alone, elastography alone and of both methods in combination with cytological results

\begin{tabular}{|c|c|c|c|c|}
\hline Imaging method & Sensitivity (\%) & Specificity (\%) & NPV (\%) & Accuracy (\%) \\
\hline $\begin{array}{l}\text { TI-RADS gray-scale score only } \\
\text { (3658 cases) }\end{array}$ & 95.7 & 61 & 99.7 & 62 \\
\hline Elastography only (991 cases) & 74.2 & 91.1 & 98 & 90 \\
\hline $\begin{array}{l}\text { Combined TI-RADS (991 cases) } \\
\text { gray-scale + elastography }\end{array}$ & 98.5 & 44.7 & 99.8 & 48.3 \\
\hline
\end{tabular}

NPV, negative predictive value. 
Table 2 Positive predictive value of the three methods compared with cytological results

\begin{tabular}{lccc}
\hline & \multicolumn{3}{c}{ Imaging method } \\
\cline { 2 - 4 } & $\begin{array}{c}\text { Gray-scale only } \\
\text { Score }\end{array}$ & $\begin{array}{c}\text { Elastography only } \\
(\% ; 958 \text { cases })\end{array}$ & $\begin{array}{c}\text { Combined gray- } \\
\text { scale+ }+ \text { clastogra- } \\
\text { phy (\%; 991 cases) }\end{array}$ \\
\hline 2 & 0 & & 0 \\
3 & 0.25 & & 0.3 \\
4A & 6 & 37.4 & 1.6 \\
4B & 69 & & 35.9 \\
5 & 100 & & 100 \\
\hline
\end{tabular}

gray-scale score only was $93.2 \%$ when compared with histopathological results and 95.1\% (194/204) when compared with the total number of carcinomas diagnosed by cytology and histology. The sensitivity of elastography only was $41.9 \%$ and of a combination of gray-scale and elastography $96.7 \%$ (Table 3). Only one carcinoma case scored TI-RADS 3 and was soft on elastography.

\section{Interobserver agreement}

For the total six-point gray-scale TI-RADS score, the $\kappa$ value was $0.72(95 \% \mathrm{CI}, 0.62-0.81)$ and Pearson's concordance correlation coefficient was 0.73 (95\% CI, 0.66-0.79). When considering only scores $4 \mathrm{~A}, 4 \mathrm{~B}$, and 5, which correspond to theoretical indications for biopsy according to the TI-RADS system, the $\kappa$ value was 0.74 (95\% CI, 0.65-0.84) and Pearson's coefficient was also 0.74 (95\% CI, 0.67-0.80).

\section{Estimation of the reduction in the number of FNABs}

Nodules with TI-RADS scores of 2 or 3 represented $52.4 \%$ of all nodules. By basing the reasoning on the figure of $35 \%$ of nodules that increase in size in time, we ended in a reduction of the number of FNABs estimated at $33.8 \%(0.65=$ scores 2 and 3 that do not grow $\times 0.524=$ percentage of nodules scored 2 and 3 ).

\section{Discussion}

Over the past two decades, widespread use of US and incidental imaging detection has contributed to an increased detection of thyroid nodules and to a threefold increase in thyroid aspirates (14). The goal to avoid unnecessary repeated US examinations and FNABs led to the development of risk stratification tools. Three other groups $(1,2,15)$ have issued reports on TI-RADS, but only one group has tested the system prospectively (1).

To detect thyroid carcinomas with gray-scale US with the best possible efficiency, we used the four US signs described by Kim et al. (16) and added solid and mildly hypoechoic as a fifth sign. The four US signs reported by Kim et al. correspond to a taller-than-wide shape, irregular borders, marked hypoechogenicity, and microcalcifications. They reported an initial sensitivity of 94\%; however, in our study, they only permitted to detect $41.1 \%$ of all carcinomas. By adding a fifth sign, the sensitivity of the TI-RADS gray-scale score was 95.7\% compared with cyto- and histopathological $(93.2 \%)$ results. To make up with the few false negative cases, which correspond to missed carcinomas, we suggest to perform FNAB when nodules initially scored TI-RADS 3 grow in a proven way during follow-up.

On the other hand, to safely rule out malignancy, we founded our score on the published material related to asserting with US that a nodule is benign $(17,18,19)$. We found a very high NPV of $99.7 \%$ for scores 2 and 3. US-TI-RADS scoring has the ability to discard malignancy with a high probability. A total of $52.4 \%$ of all nodules are classified as probably benign with a $0.3 \%$ risk of mistake.

The sensitivity of elastography was $74.2 \%$ compared with cyto- and histopathological (41.9\%) results. Specificity was 91.1 and $86.4 \%$ respectively, and accuracy 90 and $68 \%$. A hard nodule should always be thought of as highly suspicious of malignancy but low stiffness is frequently encountered in carcinomas. In our study, the sensitivity of elastography for the detection of thyroid carcinoma was lower than expected according to the meta-analysis by Bojunga et al. (20).

Table 3 Matched results of gray-scale ti-rads score and elastography with final histopathological results - (number of cases)

\begin{tabular}{|c|c|c|c|c|c|c|c|c|c|c|}
\hline & \multicolumn{5}{|c|}{ Benign histological results (130 cases) } & \multicolumn{5}{|c|}{ Malignant histological results (133 cases) } \\
\hline & \multicolumn{5}{|c|}{ Bethesda's category } & \multicolumn{5}{|c|}{ Bethesda's category } \\
\hline & 2 & 3 & 4 & 5 & 6 & 2 & 3 & 4 & 5 & 6 \\
\hline \multicolumn{11}{|c|}{ Gray-scale score only } \\
\hline TI-RADS 3 & 9 & 2 & 9 & 6 & & & 1 & 1 & 2 & 5 \\
\hline TI-RADS 4A & 1 & 3 & 65 & 26 & & & 1 & 10 & 35 & 29 \\
\hline TI-RADS 4B & & 1 & 3 & 4 & 1 & & 1 & 1 & 12 & 28 \\
\hline TI-RADS 5 & & & & & & & & & 1 & 6 \\
\hline Total & 10 & 6 & 77 & 36 & 1 & & 3 & 12 & 50 & 68 \\
\hline \multicolumn{11}{|c|}{ Elastography only } \\
\hline Soft nodule & 4 & 1 & 18 & 12 & & & 1 & 7 & 5 & 5 \\
\hline Hard nodule & & 1 & 5 & 1 & 1 & & 1 & & 2 & 10 \\
\hline Total & 4 & 1 & 23 & 13 & & & 2 & 7 & 7 & 15 \\
\hline
\end{tabular}


Most of our cases were referred for FNAB of a single nodule, therefore constituting a potential selection bias. This discrepancy in results may also be explained by the fact that the study by Bojunga et al. was largely based on qualitative elastographic techniques, different from the quantitative technique we used. In agreement with our results, Ünlütürk et al. (21) noted a lower sensitivity and specificity of US elastography for the diagnosis of malignant thyroid nodules than previously reported.

The combination of gray-scale US and elastography can be used in two separate ways. Firstly, it can increase sensitivity because it can detect a small percentage of carcinomas omitted by gray-scale alone. Trimboli et al. (22) used signs for detecting carcinomas that were identical to our group and reported a sensitivity of only $85 \%$ with gray-scale. This value increased to $97 \%$ when gray-scale was combined with elastography. However, Moon et al. (23) found that the combination of both methods showed inferior performance in the differentiation of malignant and benign thyroid nodules compared with gray-scale US features. Secondly, a combination of gray-scale US and elastography can raise specificity. Biopsying or monitoring nodules scored TI-RADS 4A with gray-scale that are soft on elastography could be discussed, since the risk of carcinoma dropped to $1.6 \%$ in our study vs $6 \%$ among all nodules that scored 4A (Table 2).

\section{Enhance interobserver agreement}

While US scanning is noninvasive, a major concern is that it is operator dependent. Regarding reproducibility, Hambly et al. (24) studied the interobserver agreement of a five-point malignancy rating scale very similar to the TI-RADS score on 101 nodules. The interobserver agreement on whether to proceed to biopsy was fair to substantial, ranging from a $\kappa$ value of $0.38-0.69$. In our study on 180 nodules, for the complete six-point scale, corresponding to the likelihood for malignancy, $\kappa$ value and Pearson's concordance correlation coefficient were respectively 0.72 and 0.73 . Considering that nodules with scores of 2 and 3 should not undergo biopsy and that scores of 4 and 5 should, the interobserver agreement for biopsy had a $\kappa$ value of 0.74 . In both cases, interobserver agreement was substantial.

\section{Rationalize the indications for FNABs and avoid unnecessary ones}

Proven high sensitivity, NPV, and interobserver agreement could allow to monitor the nodules with a TI-RADS score of 2 or 3, which represent $52.4 \%$ of all nodules, unless they increase in volume in a proven way. Considering that $\sim 35 \%$ of thyroid nodules increase with time, we ended in a reduction of the number of FNABs estimated at $33.8 \%$. A reduction in unnecessary FNABs obtained by using US scanning risk stratification has been studied in three reports. In a series of 450 nonpalpable nodules, Leenhardt et al. (25) reported that indication of US-FNAB appears judicious in centimetric or supracentimetric nodules or in solid and hypoechoic ones. This disease management would avoid $16 \%$ of unnecessary biopsies. In 2006, Cappelli et al. (5) performed a retrospective study on 6135 nodules. These authors suggested performing FNAB on nodules with the taller-than-wide sign or on those having at least two of the following three signs: microcalcifications, ill-defined borders, and hypoechogenicity. This strategy would omit $0.9 \%$ of cancers, while reducing the number of FNABs by $28 \%$. Finally, Horvath et al. in 2009 (1) stated that the indication of FNABs was useless in $34 \%$ of all cases. Among these, only $1 \%$ corresponded to carcinomas according to the cytological results.

The results of these previous reports are consistent with ours. In our practice, scoring is now used daily to select nodules for US-FNAB. Based on the French Endocrine Society consensus (26) and its indications for FNAB for nodules $>7 \mathrm{~mm}$, we suggest taking into account the importance of TI-RADS scoring and propose indications of FNAB as follows: i) between 7-10 mm, we recommend FNAB for scores of $4 \mathrm{~B}$ and 5 or when the patient has risk factors (family history of thyroid cancer, neck irradiation during childhood) or if there is focal PET-SCAN uptake and ii) $>10 \mathrm{~mm}$, we recommend $\mathrm{FNAB}$ for a score of $4 \mathrm{~A}$ or 3 that grows in a proven way (26), that is to say $2 \mathrm{~mm}$ or more in two perpendicular planes and more than $20 \%$ in volume. Of course, these are general guidelines, and from a medical and legal point of view, the indication of FNAB should always be tailored to each patient.

This study has some limitations. Firstly, several US signs are not taken into account in the flowchart, including macrocalcifications, the halo sign, and central vascularization. This was done to simplify the system and to increase interobserver agreement; however, these signs and others may be instrumental in refining one's judgment on a particular nodule. This is the counterpart of the process of systematization in that it truncates the complexity of reality. Secondly, clinical behavior, TSH assessment, and results of scintigraphy, when available, should also be taken into account for the indication of FNAB. Thirdly, the fact that very small nodules were subjected to FNAB could be disputable; however, the aim of our study was to test the TI-RADS score as independently as possible of those factors. Fourthly, this study represents a single specialized thyroid clinic's work; thus, results have to be confirmed with multicenter studies and tested by nonspecialized US practitioners. Fifthly, the decrease in the number of unnecessary biopsies was only estimated and needs a proper randomized prospective trial for itself. Finally, histological confirmation is unavailable for $53.6 \%$ of cytological cases categorized Bethesda 4 to 6. Part of this bias is compensated for by the size of the population included in this series and its prospective technique. 
In conclusion, the TI-RADS score is a six-point scale for risk stratification of thyroid nodules. It gives us the ability to detect most thyroid carcinomas and to assert benign status with good reliability for more than $50 \%$ of all nodules. Elastography can be used to raise sensitivity or specificity. Interobserver agreement is substantial. TI-RADS could lead to a significant decrease of the number of unnecessary FNABs.

\section{Declaration of interest}

The authors declare that there is no conflict of interest that could be perceived as prejudicing the impartiality of the research reported.

\section{Funding}

This research did not receive any specific grant from any funding agency in the public, commercial or not-for-profit sector.

\section{References}

1 Horvath E, Majilis S, Rossi R, Franco C, Niedmann J, Castro A \& Dominguez M. An ultrasonogram reporting system for thyroid nodules stratifying cancer risk for clinical management. Journal of Clinical Endocrinology and Metabolism 200990 1748-1751. (doi:10.1210/jc.2008-1724)

2 Park JY, Lee HJ, Jang HW, Kim HK, Yi JH, Lee W \& Kim SH. A proposal for a thyroid imaging reporting and data system for ultrasound features of thyroid carcinoma. Thyroid $2009 \mathbf{1 9}$ 1257-1264. (doi:10.1089/thy.2008.0021)

3 American College of Radiology. Breast imaging reporting and data system: BI-RADS Atlas, 4th edn. Reston, VA, 2003.

4 Russ G, Bigorgne C, Royer B, Rouxel A \& Bienvenu-Perrard M. Le système TIRADS en échographie thyrö̈dienne. Journal de Radiologie 201192 701-713. (doi:10.1016/j.jradio.2011.03.022)

5 Rago T, Vitti P, Chiovato L, Mazzeo S, De Liperi A, Miccoli P, Viacava P, Bogazzi F, Martino E \& Pinchera A. Role of conventional ultrasonography and color flow-doppler sonography in predicting malignancy in 'cold' thyroid nodules. European Journal of Endocrinology 1998138 41-46. (doi:10.1530/eje.0.1380041)

6 Cappelli C, Castellano M, Pirola I, Gandossi E, De Martino E, Cumetti D, Agosti B \& Rosei EA. Thyroid nodule shape suggests malignancy. European Journal of Endocrinology 2006155 27-31. (doi:10.1530/eje.1.02177)

7 Park SH, Kim SJ, Kim E-K, Kim MJ, Son EJ \& Kwak JY. Interobserver agreement in assessing the sonographic and elastographic features of malignant thyroid nodules. American Journal of Roentgenology 2009193 416-423. (doi:10.2214/AJR.09.2541)

8 Cibas ES \& Ali SZ. The Bethesda system for reporting thyroid cytopathology. Thyroid 200919 1159-1165. (doi:10.1089/thy. 2009.0274)

9 Brander AE, Viikinkoski VP, Nickels JI \& Kivisaari LM. Importance of thyroid abnormalities detected at US screening: a 5-year followup. Radiology 2000215 801-806.

10 Kuma K, Matsuzuka E, Yokozawa T, Miyauchi A \& Sugawara M. Fate of untreated benign thyroid nodules: results of longterm follow-up. World Journal of Surgery $1994 \mathbf{1 8} 495-498$. (doi:10.1007/BF00353745)

11 Cheung PSY, Lee JMH \& Boey JH. Thyroxine suppressive therapy of benign solitary thyroid nodules: a prospective randomized study. World Journal of Surgery 198913 818-822. (doi:10.1007) BF01658447)
12 Alexander EK, Hurwitz S, Heering JP, Benson CB, Frates MC, Doubilet PM, Cibas ES, Larsen PR \& Marqusee E. Natural history of benign solid and cystic thyroid nodules. Annals of Internal Medicine $2003138315-318$.

13 Russ G, Royer B, Bigorgne C \& Rouxel A. Real-time quantitative ultrasound elastography of thyroid nodules. International Thyroid Congress 2010 (Abstract P-0377).

14 Cronan JJ. Thyroid nodules: is it time to turn off the US machines? Radiology $2008 \mathbf{2 4 7}$ 602-604. (doi:10.1148/radiol. 2473072233)

15 Kwak JY, Han KH, Yoon JH, Moon HJ, Son EJ, Park SH, Jung HK, Choi JS, Kim BM \& Kim E-K. Thyroid imaging reporting and data system for US features of nodules: a step in establishing better stratification of cancer risk. Radiology 2011260 892-899. (doi:10.1148/radiol.11110206)

16 Kim EK, Park CS, Chung WY, Oh KK, Kim DI, Lee JT \& Yoo HS. New sonographic criteria for recommending fine needle aspiration biopsy of non palpable solid nodules of the thyroid. American Journal of Roentgenology $2002 \mathbf{1 7 8} 687-691$.

17 Reading CC, Charboneau JW, Hay ID \& Sebo TJ. Sonography of thyroid nodules: a "classic pattern" diagnostic approach. Ultrasound Quarterly 200521 157-165. (doi:10.1097/01.ruq. $0000174750.27010 .68)$

18 Bonavita JA, Mayo J, Babb J, Bennett G, Oweity T, Macari M \& Yee J. Pattern recognition of benign nodules at ultrasound of the thyroid: which nodules can be left alone? American Journal of Roentgenology 2009193 207-213. (doi:10.2214/AJR.08.1820)

19 Virmani V \& Hammond I. Sonographic patterns of benign thyroid nodules: verification at our institution. American Journal of Roentgenology 2011196 891-895. (doi:10.2214/AJR.10.5363)

20 Bojunga J, Herrmann E, Meyer G, Weber S, Zeuzem S \& Mireen F-R. Real-time elastography for the differentiation of benign and malignant thyroid nodules: a meta-analysis. Thyroid $2010 \mathbf{2 0}$ 1145-1150. (doi:10.1089/thy.2010.0079)

21 Ünlütürk U, Erdoğan MF, Demir O, Güllü S \& Başkal N. Ultrasound elastography is not superior to gray-scale ultrasound in predicting malignancy in thyroid nodules. Thyroid 201222 1031-1038. (doi:10.1089/thy.2011.0502)

22 Trimboli P, Guglielmi R, Monti S, Misischi I, Graziano F, Nasrollah N, Amendola S, Morgante SN, Deiana MG, Valabrega $S$ et al. Ultrasound sensitivity for thyroid malignancy is increased by real-time elastography: a prospective multicenter study. Journal of Clinical Endocrinology and Metabolism 201297 4524-4530. (doi:10.1210/jc.2012-2951)

23 Moon HJ, Sung JM, Kim E-K, Yoon JH, Youk JH \& Kwak JY. Diagnostic performance of gray-scale US and elastography in solid thyroid nodules. Radiology 2012262 1002-1013. (doi:10.1148/ radiol.11110839)

24 Hambly NM, Gonen M, Gerst SR, Li D, Jia X, Mironov S, Sarasohn D, Fleming SE \& Hann LE. Implementation of evidence-based guidelines for thyroid nodule biopsy: a model for establishment of practice standards. American Journal of Roentgenology 2011196 655-660. (doi:10.2214/AJR.10.4577)

25 Leenhardt L, Hejblum G, Franc B, Fediaevski LD, Delbot T, Le Guillouzic D, Ménégaux F, Guillausseau C, Hoang C, Turpin G et al. Indications and limits of ultrasound-guided cytology in the management of nonpalpable thyroid nodules. Journal of Clinical Endocrinology and Metabolism $1999 \mathbf{8 4} 24-28$. (doi:10.1210/jc. 84.1.24)

26 Wémeau JL, Sadoul J-L, d'Herbomez M, Monpeyssen H, Tramalloni J, Leteurtre E, Borson-Chazot F, Caron P, Carnaille B, Léger J et al. Guidelines of the French society of endocrinology for the management of thyroid nodules. Annales d'Endocrinologie 2011 72 251-281. (doi:10.1016/j.ando.2011.05.003)

Received 25 October 2012

Revised version received 26 January 2013

Accepted 14 February 2013 